\title{
PO3-13-20
}

Poster session

\section{Cohort study of liver toxicity of azole antifungal medication in a real-life hospital setting}

\section{Tonny S Petersen, Kim P Dalhoff}

\section{Clinical Pharmacology, Bispebjerg and Frederiksberg University Hospital, Copenhagen, Denmark}

Background: Azole antifungal medications are known to cause liver toxicity based on several clinical trials, which, however, may not represent real life patients due to exclusion criteria in the studies. Observational studies in a real life hospital setting are lacking, and we therefore investigated the prevalence of liver toxicity in the hospitals in the Capital Region of Denmark.

Methods: We identified patients from the electronic health records treated with at least one administration of an azole antifungal administered by the hospital between January 2010 and May 2016 and which had at least one recent pretreatment and one on-treatment measurement of ALT. For each treatment period we extracted information about dose, maximum ALT, and comorbidity.

We defined liver toxicity as an increase in ALT of 3 times the upper normal level or baseline whichever where highest. Results: We identified 52,103 treatment-periods in which an azol antifungal drug was used; of these 19,954 had sufficient ALTs for inclusion. $52 \%$ of the cases had a recent cancer diagnosis. 4,380 cases (22\%) had an abnormal pre-treatment ALT. Totally 660 cases (3.3\%) had signs of liver toxicity, which was more commonly observed in patients with increased pre-treatment ALT (4.4\%). The distribution of liver toxicity among the azoles was as follows: miconazole $(72 / 2,886$; $2.5 \%)$, ketoconazole $(1 / 5 ; 20 \%)$, fluconazole $(487 / 14,636 ; 3.3 \%)$, itraconazole $(5 / 109 ; 4.6 \%)$, voriconazole $(43 / 807$; $5.3 \%)$, posaconazole $(52 / 1,511 ; 3.4 \%)$. Multiple logistic regression analysis revealed several risk factors for developing liver toxicity: preexisting liver disease (OR: 1.6; 95\% CI: 1.0-2.5), elevated pre-treatment ALT (OR: 1.5; 95\% CI: 1.3 1.8), high dose (OR increased 1.3 times per DDD increase in dose; 95\% CI: 1.2 - 1.5). A diagnosis of alcohol dependency was associated with a lower risk of developing liver toxicity (OR: 0.47; 95\% CI: $0.27-0.74$ ). Only voriconazole was associated with an increased risk of developing liver toxicity compared to fluconazole (OR: 1.7; 95\% CI: 1.2 - 2.3).

Conclusion: The prevalence of liver toxicity was in line with previous reports. Voriconazole was associated with a higher prevalence. The unexpected finding of a lower prevalence of liver toxicity among alcohol dependent patients warrants further investigations. 